OPEN ACCESS

Edited by:

José Manuel Pérez-Pérez,

Universidad Miguel Hernández

de Elche, Spain

Reviewed by:

Abhishek Bohra,

Indian Institute of Pulses Research,

Dong-Yup Lee,

National University of Singapore,

Singapore

*Correspondence:

Xinghua Wei

weixinghua@caas.cn

${ }^{\dagger}$ These authors have contributed equally to this work.

Specialty section:

This article was submitted to Plant Genetics and Genomics,

a section of the journal

Frontiers in Plant Science

Received: 13 December 2016

Accepted: 13 April 2017

Published: 25 April 2017

Citation:

Zhang $M, L u$ Q, Wu W, Niu X Wang $C$, Feng $Y, X u Q$, Wang $S$, Yuan $X$, Yu $H$, Wang $Y$ and Wei $X$

(2017) Association Mapping Reveals

Novel Genetic Loci Contributing to Flooding Tolerance during

Germination in Indica Rice. Front. Plant Sci. 8:678. doi: 10.3389/fpls.2017.00678

\section{Association Mapping Reveals Novel Genetic Loci Contributing to Flooding Tolerance during Germination in Indica Rice}

\author{
Mengchen Zhang ${ }^{1 t}$, Qing Lu',2t, Wei Wu ${ }^{3}$, Xiaojun Niu' ${ }^{1}$, Caihong Wang ${ }^{1}$, Yue Feng ${ }^{1}$, \\ Qun $\mathrm{Xu}^{1}$, Shan Wang ${ }^{1}$, Xiaoping Yuan ${ }^{1}$, Hanyong Yu${ }^{1}$, Yiping Wang ${ }^{1}$ and Xinghua Wei ${ }^{1 *}$ \\ 1 State Key Laboratory of Rice Biology, China National Rice Research Institute, Hangzhou, China, ${ }^{2}$ Crop Research Institute, \\ Guangdong Academy of Agricultural Sciences (GAAS), South China Peanut Sub-Center of National Center of Oilseed Crops \\ Improvement, Guangdong Key Laboratory for Crops Genetic Improvement, Guangzhou, China, ${ }^{3}$ Seeds Administration \\ Center of Zhejiang Province, Hangzhou, China
}

Rice (Oryza sativa L.) is the only cereal crop that possesses the ability to germinate under flooded or other oxygen-deficient conditions. Rapid elongation of the coleoptile is a perfect response to flooding during germination, with coleoptile length differing among various rice varieties. Despite multiple studies have uncovered valuable information concerning this trait by focusing on the physiological metabolism of oxygen stress, the underlying genetic mechanism still remains unknown. In the present study, we screened coleoptile lengths of 432 indica varieties germinated in two environments (normal and flooded) and found more variation existing in flooded coleoptile length (FCL) rather than in normal coleoptile length (NCL). With the phenotypic data of NCL, FCL and FTI (flooding tolerance index), a genome-wide association study was performed by using 5291 single nucleotide polymorphism (SNP) markers. We detected 2, 11, and 9 significant SNPs under a mixed linear mode for NCL, FCL, and FTI, respectively. Of these SNPs, five were shared by FCL and FTI. Haplotype and phenotype effect analysis on the highest ranking locus indicated one of the two haplotypes contributed to coleoptile elongation remarkably. To better understand the controlling gene of this locus, reported expression profile data was applied. We focused on LOC_Os06g03520, a candidate gene which was highly induced by anoxia ( 507 fold). Sequence analysis in 51 varieties demonstrated Hap.2 associated perfectly with flooding tolerance. Further studies on this gene may help explore the molecular mechanism of rice flooding tolerance during germination. We believe our discoveries may conduce to isolating major genes and aid the improvement of flooding tolerance in modern breeding programs.

Keywords: genome-wide association study (GWAS), coleoptile, flooding, germination, rice (Oryza sativa L.)

\footnotetext{
Abbreviations: FCL, flooded coleoptile length; FTI, flooding tolerance index; GWAS, genome-wide association study; LD, linkage disequilibrium; MLM, mixed linear model; NCL, normal coleoptile length; PCA, principal component analysis; QTL, quantitative trait locus; SNP, single nucleotide polymorphism.
} 


\section{INTRODUCTION}

Flooding is a widespread, recurring problem that negatively affect rice (Oryza sativa L.) production in rain-fed lowlands areas of south and south-east Asia (Septiningsih et al., 2009). To respond to the oxygen-deficient stress caused by flooding, rice coleoptile develops fast to reach the surface of flooding water thus allow oxygen to transport to underwater tissues (Alpi and Beevers, 1983; Magneschi et al., 2009). This response is likely to be one of the most important patterns to study crop anaerobic metabolism.

Submergence caused by flooding creates a hypoxic environment that restricts plant aerobic respiration and leads to low ATP production. Originating in a semi-aquatic environment, rice has evolved a complicated response mechanism to adapt to long periods of submergence (Nagai et al., 2010; Kudahettige et al., 2011). The establishment of coleoptile at germination stage and highly developed aerenchyma in mature plants allow rice tissues to transport oxygen under water. Physiologically, various metabolic changes take place during flooding or oxygen-deficiency conditions. Enzymes related to starch degradation, glycolysis and ethanol fermentation, such as $\alpha$-amylases, phosphofructokinase, fructose-6-phosphate-1phosphotransferase, alcohol dehydrogenase and pyruvate dehydrogenase, are highly active under submerged conditions (Gibbs et al., 2000; Kato-Noguchi and Morokuma, 2007; Lasanthi-Kudahettige et al., 2007; Magneschi and Perata, 2009). Glucose also plays a positive role in flooding tolerance, with the exogenous application shown to restore this trait in intolerant type (Huang et al., 2003; Loreti et al., 2005). As a consequence, sugar and ATP concentrations can be maintained to provide basic energy for survival. In particularly, the high expression of $R A M Y 3 D$ and $A D H$ correlated with the tolerance of flooding - an observation supported by numerous studies (Kato-Noguchi and Morokuma, 2007; Lasanthi-Kudahettige et al., 2007; Magneschi et al., 2009).

Genetic studies on rice germination under oxygen-deficiency condition mainly focus on exploring the controlling genes and their functional mechanisms. Numerous QTLs have been detected by using different genetic mapping populations. Angaji (2008) reported four putative QTLs on chromosomes 1, 2, 11 , and 12 in a $\mathrm{BC}_{2} \mathrm{~F}_{2}$ population derived from the cross of KHAIYAN and IR64. These QTLs explained 51.4\% of the phenotypic variance. Similarly, Khao Hlan On, a tolerant accession, was crossed with IR64 to construct $\mathrm{BC}_{2} \mathrm{~F}_{2}$ lines, and five QTLs were found on chromosomes 1, 3, 7, and 9 (Angaji et al., 2009). Septiningsih et al. (2013) unraveled six significant QTLs on chromosomes 2, 5, 6, and 7 by using a $\mathrm{F}_{2: 3}$ population derived from IR42 and Ma-Zhan Red. These genetic loci would be valuable for further genetic mechanism researches.

Among the cloned genes related to flooding tolerance, Sub1A, one of the three ethylene-responsive factor (ERF) genes at Sub1 locus, enables mature rice plants to survive 10-14 days under complete submergence stress (Fukao et al., 2006; Xu et al., 2006). With submergence, Sub1A significantly increases the GA signaling repressors Slender Rice-1 (SLR1) and SLR1 Like-1 (SLRL1) to suppress the GA-mediated growth regulation and diminishes other GA induced genes expression (Fukao and Bailey-Serres, 2008). Although Sub1A has major effect on submergence tolerance of mature rice plant, some rice cultivars lacking this locus, such as Nipponbare, display typical response to submergence during germination (Magneschi and Perata, 2009). This tolerance suggests that a Sub1A-independent mechanism may exist during rice germination under submergence (Lee et al., 2009). $\mathrm{Ca}^{2+}$ is a signal transducer during hypoxia and is also required for enzymes associated with glycolytic and ethanol fermentation in maize and Arabidopsis (Subbaiah et al., 1994; Sedbrook et al., 1996). The Calcineurin B-like (CBL) interacting protein kinases (CIPKs) has been found to act as key regulators of energy supply when oxygen availability is restricted, thus proving that $\mathrm{Ca}^{2+}$ signals play important roles in response to the oxygen stress (Batistic and Kudla, 2004). OsCIPK15, a CIPK family member, whose encoding gene is located on rice chromosome 12 , functions in the sensing cascade associated with rice response to oxygen-deficiency (Lee et al., 2009). In rice, CIPK15 positively regulates sucrose nonfermenting 1 related protein kinase (SnRK1A) which is an essential regulator of MYBS1 (Halford et al., 2003; Lu et al., 2007). MYBS1 interacts with the sugar response complex (SRC) at the promoters of $\alpha$-amylase encoding genes ( $\mathrm{Lu}, 2002$; Chen et al., 2006). The CIPK15-SnRK1A-MYBS1- $\alpha$-amylase signaling pathway is suggested to be a critical response to sugar starvation when seeds suffer from submergence. OsCIPK15 is also involved in the growth of the mature rice plants subjected to partial flooding (Lee et al., 2009; Kudahettige et al., 2011). OsTPP7, a gene encodes trehalose-6-phosphate phosphatase, was recently reported to enhance anaerobic germination tolerance in rice (Kretzschmar et al., 2015). Although the signal pathway of OsTPP7 remains unclear at present, studies revealed that OsTPP7 was involved in trehalose-6-phosphate metabolism and functions in enhancing starch mobilization to increase the tolerance of anaerobic germination.

With the first report of uncovering 14 agronomic traits in rice landraces, GWAS has become a popular strategy to dissect complex traits in rice (Huang et al., 2010). Compared with linkage mapping approach, association mapping possesses the ability to uncover a larger number of superior allele variations in broad natural populations, simultaneously in a simple and rapid pattern. Even though most published reports failed to identify new controlling gene, Yano et al. (2016) reported four novel genes influencing rice heading date, plant height, panicle number per plant and awn length with a single association study. These achievements rise new hope of uncovering novel genes with association mapping approach.

As the only organic response against oxygen-deficient stress, coleoptile elongation has become a reliable trait to study flooding tolerance of rice genotypes (Setter, 1994; KatoNoguchi and Morokuma, 2007; Magneschi et al., 2009). In the present study, GWAS was performed on an indica population using reported SNP data ( $\mathrm{Lu}$ et al., 2015), with respect to rice coleoptile elongation under normal and flooded environments. The objectives of this study were to: (a) detect the major genetic loci controlling rice flooding tolerance during germination; (b) explore favorable SNP alleles potentially useful 
for breeding; (c) find the most likely candidate gene. The results provide basic theory to enhance flooding tolerance in breeding program and also enable us to explore the genetic mechanism behind.

\section{MATERIALS AND METHODS}

\section{Plant Materials and Germination Experiment}

On the basis of a previous study, we selected 432 indica accessions originating from 19 countries with 5291 SNPs genotyped by a custom-designed array ( $\mathrm{Lu}$ et al., 2015) (Supplementary Table S1). Rice seeds were harvested in $\mathrm{HZ}\left(\mathrm{N} 30^{\circ} 15^{\prime}, \mathrm{E} 120^{\circ} 12^{\prime}\right)$, China, air-dried naturally, and maintained at $55^{\circ} \mathrm{C}$ for 5 days to break dormancy. Then seeds were surface-sterilized with $70 \%$ ethyl alcohol and washed three times with sterile water. For normal germination (control), plump seeds were wrapped in wet absorbent filter paper and vertically placed in a plastic box. For flooded germination, seeds were placed in a germination pouch and then submerged in sterile water to a $10-\mathrm{cm}$ depth in a plastic box $\left(\mathrm{O}_{2}\right.$ concentration was $\left.0.12-0.13 \mathrm{~mol} \mathrm{~m}^{-3}\right)$. Germination was allowed to proceed at $30^{\circ} \mathrm{C}$ in darkness for 10 days in an artificial climate chamber. Three replicated experiments were implemented for both control and flooded germination. After germination, coleoptile length was measured and data was accurate to millimeter.

\section{Statistical Analysis of Phenotypic Variation}

The flooding tolerance index (FTI) was calculated as formula: flooded coleoptile length (FCL)/normal coleoptile length (NCL). Descriptive statistical analysis was performed in Microsoft Office Excel 2007. The analysis of variation (ANOVA) was carried out to evaluate the effects of genotype $(G)$, and genotype $\times$ environment $(G \times E)$ using general linear model under SAS 9.0 (SAS, Inc., Cary, NC, USA). Variation and correlation analysis (SAS 9.0) were performed respectively to evaluate the coefficient of variation (CV) and estimate the relationship between NCL and FCL.

\section{Population Structure, Kinship Estimation}

Principal component analysis was performed based on Nei's genetic distance using Powermarker (ver. 3.25) (Liu and Muse, 2005) and NTSYSpc (ver. 2.1) (Rohlf, 2000). Pairwise relative kinship coefficients were calculated in TASSEL (ver. 4.0) (Bradbury et al., 2007).

\section{Association Mapping}

Genome-wide association mapping was performed using TASSEL ver. 4.0 (Bradbury et al., 2007), and the EMMA (Kang et al., 2008) and P3D (Zhang et al., 2010) algorithms were used to reduce computing time. SNPs with a minimum count less than $75 \%$ of 432 individuals or with a minor allele frequency (MAF) less than 0.05 were removed from the association panel. Association analysis was implemented under a MLM which could be described as: $y=X \alpha+P \beta+K \mu+e$, while $X$ and $y$ were vectors of genotype and phenotype data, $\alpha$ was the SNP effects, $\beta$ represented the effects of population structure, $\mu$ was the vector of kinship background effects, e corresponded to residual effects, $P$ was the PCA matrix relating $y$ to $\beta, K$ represented the related kinship matrix (Wen et al., 2014). The first three PCs were used to construct the $P$ matrix in GWAS model.

\section{Gene Prediction and Verification}

The LD heatmap around the significant SNPs in GWAS was constructed using the R package "LDheatmap" (Shin et al., 2006) and the candidate region was estimated using $r^{2}>0.6$ (Yano et al., 2016). Candidate genes were predicted by screening the reference genome ${ }^{1}$. Online public expression profile data representing different induction of anoxic germination and aerobic germination were obtained from Rice eFP Brower of Bio-Analytic Resource for Plant Biology (BAR) database ${ }^{2}$. For qRT-PCR module, total RNA was extracted from rice coleoptiles after germination for 2 or 6 days using a MiniBEST Plant RNA Extraction kit (Takara Bio, Inc., Japan). First-strand cDNA was synthesized using PrimeScript RT Master Mix (Takara Bio Inc., Japan). To ensure cDNA quality following reverse transcription, a pair of primers straddling intron was used to detect genomic DNA. Then qPCR was performed in a twostep reaction using SYBR Premix Ex TaqII (Tli RNase Plus) (Takara Bio, Inc., Japan) on an Applied Biosystems 7500 RealTime PCR system (Applied Biosystem, Carlsbad, CA, USA) with a pair of specific primers (CD3-f: atcaccttcaaccgccacat; CD3-r: gcggagtccatgtccatctg).

The full length of candidate gene and its promoter region were amplified by polymerase chain reaction (PCR) with two pairs of specific primers (QS14-f: tagatggatgagcacgcagc; QS14r: gttcccctgcacaaacaac; QS15-f: gtaccacgaccaccatcttga; QS15-r: tgagctgatctcttggtcacg). Then PCR productions were purified and sequenced. All the sequences were edited and assembled using SeqMan in the package DNASTAR Lasergene (DNASTAR, Inc., Madison, WI, USA). Sequence analysis was performed using software MEGA6 (Tamura et al., 2013) and the haplotype analysis was carried out using DNAsp v5 (Librado and Rozas, 2009).

\section{RESULTS}

\section{Statistical Analysis of Phenotypic Variation}

Totally, 432 indica varieties were screened for NCL and FCL (Supplementary Table S1). Coleoptile length ranged from 0.98 to $4.52 \mathrm{~cm}$, with a mean value of $2.08 \mathrm{~cm}$ under normal germination condition (control). While the data ranged from 0 to $3.15 \mathrm{~cm}$, with a mean value of $1.27 \mathrm{~cm}$ under flooded germination condition. The ANOVA indicated that both Genotype and Environment had significant effects on coleoptile lengths (Table 1). Moreover, the CV was 0.22 for NCL and 0.52

\footnotetext{
${ }^{1}$ http://rice.plantbiology.msu.edu/

${ }^{2}$ http://142.150.215.220/
} 
for FCL, revealing that more abundant variation existed in FCL. The correlation analysis indicated that there was merely low relationship between NCL and FCL $\left(R^{2}=0.06, P=0.2360\right)$. The FTI was calculated according to the ratio of phenotypic data of two environments. The results presented in Table 1 showed that FTI ranged from 0 to 1.59 , with a mean value of 0.65 . The CV was 0.54 , which was even higher than that of FCL.

\section{Population Structure and Relative Kinship}

Considering that population structure may still exist in indica varieties and lead to false associations, we performed PCA based on Nei's genetic distances (Supplementary Table S2). Figure 1A displayed genetic variation explained by the top 10 principle components (PCs) in bar chart. Most PCs explained very small proportion of genetic variation except the first one, suggesting a weak genetic structure of our population. According to our calculations, most varieties had no meaningful relationship except $2.5 \%$ of varieties possessed the kinship value larger than 0.5 , which indicated the relative kinship of our population might have no strong influence on GWAS (Figure 1B). The analysis of genetic structure and relative kinship made it clear that our indica population was qualified for GWAS.

\section{Genome-Wide Association Mapping}

Association mapping was performed under MLM with PC matrix and kinship matrix as covariates. At the thresholds of $P=0.001$,

\begin{tabular}{lcccc}
\hline \multicolumn{4}{l}{ TABLE 1 | Statistical analysis of rice coleoptile length. } \\
\hline Environments & Mean $(\mathbf{c m}) \pm$ SE & Range & CV & G $\times$ E \\
\hline NCL & $2.08 \pm 0.001$ & $0.98 \sim 4.51(\mathrm{~cm})$ & 0.2155 & $* * * *$ \\
FCL & $1.27 \pm 0.001$ & $0.00 \sim 3.15(\mathrm{~cm})$ & 0.5248 & \\
FII & $0.65 \pm 0.0008$ & $0 \sim 1.60$ & 0.5367 &
\end{tabular}

$\mathrm{CV}$, coefficient of variation; NCL, normal coleoptile length; FCL, flooded coleoptile length; FTl, flooding tolerance index. ${ }^{* * *} P<0.001$. we detected totally 22 significant SNP loci associated with NCL, FCL, and FTI (Figure 2 and Table 2). Only two SNPs were detected under NCL, which were much less than the others. Moreover, none of these two SNPs were detected in the other two associations. These results confirmed that no correlation existed between the coleoptile length observed in control and flooded environments. The comparison of association between FCL and FTI identified five common SNPs, including two SNPs on chromosome 6, two SNPs on chromosome 7 and one SNP on chromosome 11 (Figure 2). These consistent loci revealed the similar contribution of FCL and FTI. The physical distance of two significant SNPs on chromosome 6 was only $211-\mathrm{kb}$, which suggested that they might belong to the same genetic loci. Considering its contribution to $P$-values and the steady identification by both FCL and FTI, this locus should be regarded as a major controller of rice flooded germination tolerance.

To verify the accuracy of our results, we compared significant SNP loci in this study with reported QTLs which were detected previously using linkage or association mapping approach. As a consequence, some of our associated loci were not first detected in the research field of rice flooded germination tolerance (Table 2). The significant SNP seq-rs3210 on chromosome 7 in our study was found to locate closely to a genetic locus which was detected in another association study (Hsu and Tung, 2015). On chromosome 7, another significant SNP seqrs3583 was identified in the genomic interval of qAG-7-2. This locus was also detected using both linkage mapping and association mapping approach (Angaji et al., 2009; Hsu and Tung, 2015), confirmed its real genetic effect. On chromosome 11, associated SNP seq-rs5120 was mapped in the genomic region of qAG-11 (Jiang et al., 2006; Angaji et al., 2009). In addition, SNP seq-rs4216, which was detected only in FTI, shared a very short distance with OsTPP7 (Kretzschmar et al., 2015). Even though some of the co-localized genetic loci were previously detected using survival rates as the phenotypic indicator, they were also detected using FCL or FTI in this study.
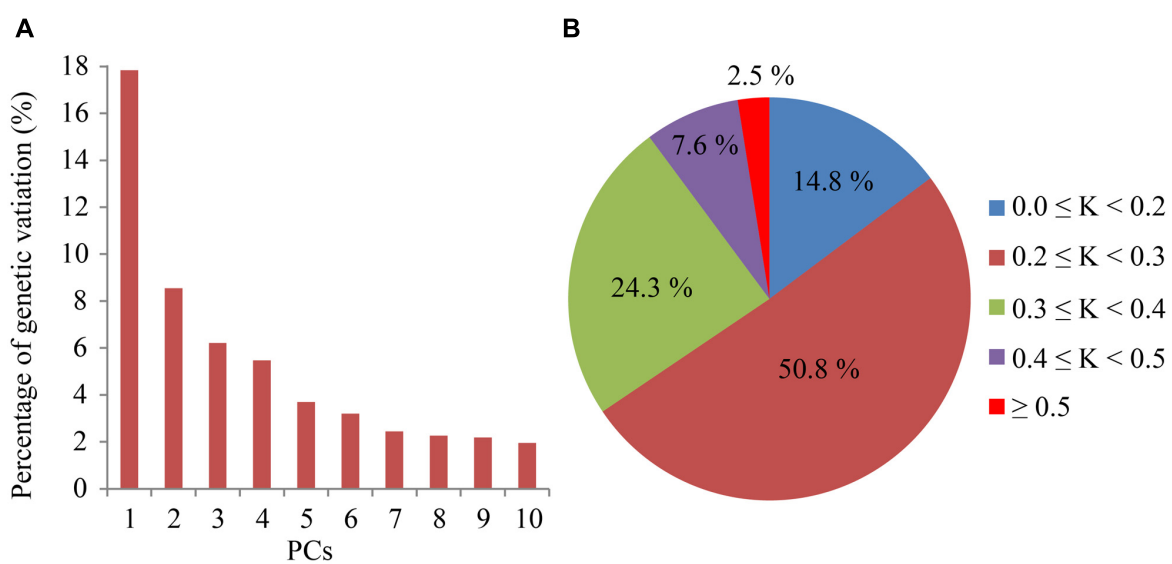

FIGURE 1 | Principal component analysis and relative kinship analysis of the indica population. (A) The percentage of genetic variation explained by each of the first 10 principal components (PCs). (B) Distribution of relative kinship among 432 indica accessions, $\mathrm{K}$ represents relative kinship coefficients. 

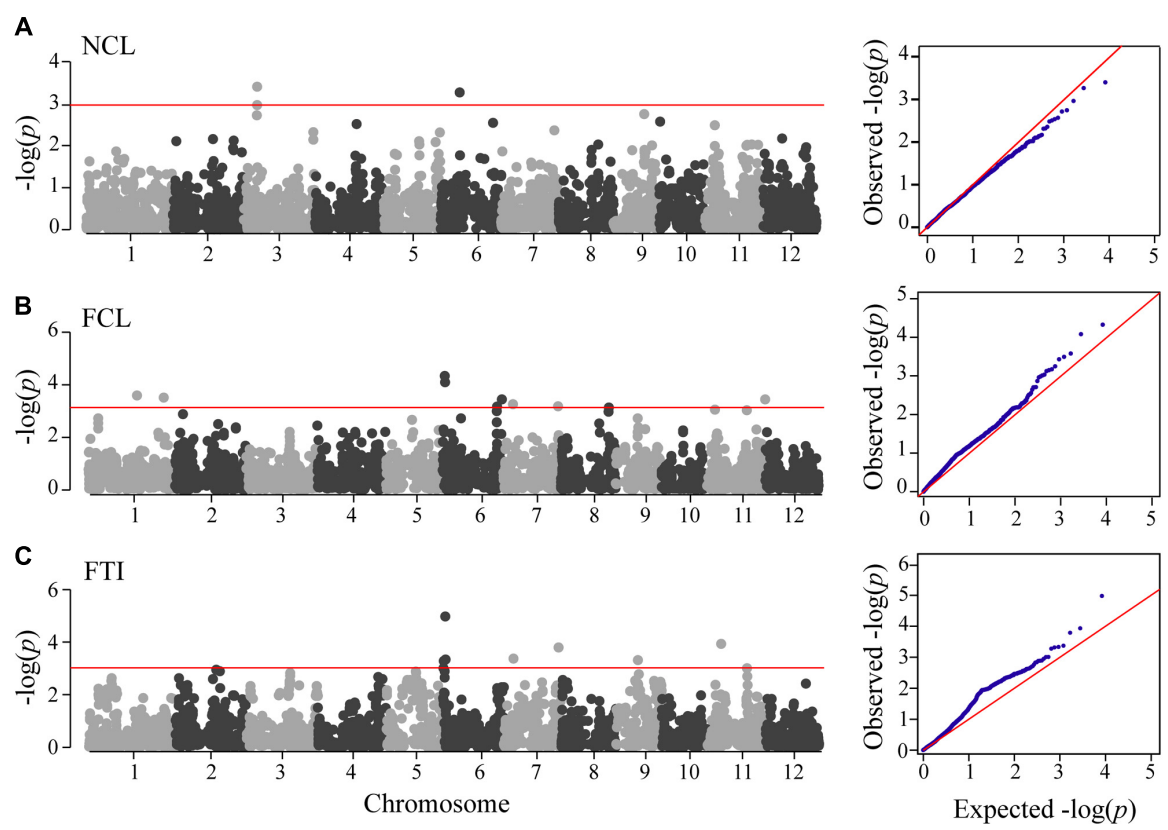

FIGURE 2 | Manhattan plots and quantile-quantile plots of genome-wide association analysis. Manhattan plots, the red straight line show the threshold of $P=0.001$; quantile-quantile plot, the red straight line represent the expected null distribution of $P$-values, the blue dots represent the observed distribution of $P$-values. (A) NCL, normal coleoptile length; (B) FCL, flooded coleoptile length; (C) FTI, flooding tolerance index.

TABLE 2 | Summary of significant SNPs detected by GWAS.

\begin{tabular}{|c|c|c|c|c|c|c|}
\hline Traits & SNP markers & $P$-value & Chromosome & IRGSP.v4 position & IRGSP 1.0 position & Known loci \\
\hline NCL & seq-rs2895 & $5.50 \mathrm{E}-04$ & 6 & 10197319 & 10198318 & \\
\hline $\mathrm{FCL}$ & seq-rs583 & 3.25E-04 & 1 & 39044291 & 37288444 & Hsu and Tung, 2015 \\
\hline FCL & seq-rs2699 & 4.75E-05 & 6 & 1178093 & 1179092 & \\
\hline FCL & seq-rs2701 & 8.34E-05 & 6 & 1389722 & 1390721 & \\
\hline $\mathrm{FCL}$ & seq-rs3210 & 5.70E-04 & 7 & 5014710 & 4982558 & Hsu and Tung, 2015 \\
\hline FCL & seq-rs3583 & $6.81 \mathrm{E}-04$ & 7 & 28544962 & 27884804 & qAG-7-2, Angaji et al., 2009; Hsu and Tung, 2015 \\
\hline FCL & seq-rs3972 & $7.58 \mathrm{E}-04$ & 8 & 24927836 & 24839831 & qAG-8-1, Angaji et al., 2009 \\
\hline FCL & seq-rs4773 & 9.33E-04 & 11 & 4581585 & 4598375 & \\
\hline FCL & seq-rs5125 & 9.81E-04 & 11 & 21084520 & 19256661 & qAG-11, Jiang et al., 2006; Angaji et al., 2009 \\
\hline FTI & seq-rs2683 & $9.91 \mathrm{E}-04$ & 6 & 276836 & 277836 & \\
\hline FTI & seq-rs3583 & $1.64 \mathrm{E}-04$ & 7 & 28544962 & 27884804 & qAG-7-2, Angaji et al., 2009; Hsu and Tung, 2015 \\
\hline $\mathrm{FTl}$ & seq-rs3210 & 4.30E-04 & 7 & 5014710 & 4982558 & Hsu and Tung, 2015 \\
\hline FTI & seq-rs4216 & 4.88E-04 & 9 & 11439838 & 10874127 & qAG-9-2, Kretzschmar et al., 2015 \\
\hline FTI & seq-rs4859 & 1.17E-04 & 11 & 7588747 & 7586818 & \\
\hline $\mathrm{FTl}$ & seq-rs5125 & 9.96E-04 & 11 & 21084520 & 19256719 & qAG-11, Jiang et al., 2006; Angaji et al., 2009 \\
\hline
\end{tabular}

\section{Analysis of Candidate Gene}

The highest ranking of SNP, seq-rs 2699 on the beginning position of chromosome 6, was firstly found to control rice FCL. In our study, this SNP was detected under both FCL and FTI. Detailed analysis on this locus was conducted to investigate the genetic mechanism behind. There were two significant SNPs on this locus which suggested the theoretic haplotypes should be at least four types. However, statistical analysis indicated that only 
two haplotypes accounted for most varieties (412 of the total 432 accessions) (Figure 3A). This result demonstrated the close linkage of these two significant SNPs. Subsequent calculation indicated phenotypic effect of these two haplotypes differed significantly. As was shown in Figure 3B, Hap.B associated with tolerant types significantly (with larger FCL and FTI). To accurately estimate the linkage region on the genomic map, pairwise LD correlations were calculated. With $r^{2}=0.6$ as the threshold, we obtained a 875 -kb candidate block containing seqrs2699 and seq-rs2701 (Figure 3C). According to the reference genome, there were 158 genes in this region enabling us to isolate the most likely candidate gene.

To narrow down the candidate gene numbers, we took advantage of a previously reported expression profile which was obtained from a japonica cultivar with respect to normal and anoxic germination (Lasanthi-Kudahettige et al., 2007). With the use of these data we focused on a candidate gene, LOC_Os06g03520, which was annotated as DUF domain containing protein. The expression level of this gene was 507 times higher in coleoptile grown in anoxic environment than in which was grown in normal environment according to the expression profile. We then detected the relative expression level in flooded coleoptiles of four tolerant indcia varieties with normal coleoptiles as control (Supplementary Figure S1). The results observed in both four indica varieties, that LOC_Os06g03520 was highly expressed in flooded coleoptiles but not in normal coleoptiles after germination for 2 or 6 days, suggested its potential possibility for controlling rice FCL (Supplementary Figure S1).

We then sequenced the full length and promoter region of LOC_Os06g03520 in varieties with extreme phenotypes (Supplementary Table S3). Totally, 27 polymorphism sites were identified including five sites located in exon regions (Figure 4A). Haplotype analysis revealed 11 haplotypes among these accessions (Figure 4B). To our surprise, almost every variety of Hap.2 was defined as tolerant type (with larger FCL and FTI) (Figures 4C,D and Supplementary Table S3), while the other haplotypes were mostly related to intolerant phenotypes. This result clearly demonstrated Hap. 2 had a strong correlation relationship with flooding tolerance. Taken together, LOC_Os06g03520 was the most likely candidate gene controlling rice flooding tolerance during germination.

\section{DISCUSSION}

\section{Diverse Phenotypic Variation in Indica Population}

One of the great necessities of studying flooding tolerance in indica subspecies is that most indica varieties are planted in low latitudes areas where they are more likely to suffer from flooding. The most sustainable methods to protect rice yields in face of such disasters is the use of flooding-tolerant cultivars (Angaji et al., 2009). We performed GWAS on an indica population and tried to detect the functional loci which could potentially use for breeding. Similar research has been reported before but with very small number of varieties and also lacking the evidence for targeted genes (Hsu and Tung, 2015). Our study with diverse indica varieties may enhance our knowledge for indica flooding tolerance. Previous researches reported that flooded coleoptiles of japonica varieties elongated faster than indica varieties (LasanthiKudahettige et al., 2007; Hsu and Tung, 2015). However the difference of flooding tolerance inside indica subspecies still remains unknown. Our finding indicated varieties responded diversely to flooded germination in indica subspecies. This diversity would be beneficial to explore the genetic mechanism of flooding tolerance during indica rice germination.

Coleoptile elongation has been regarded as a major response to anaerobic stress (Setter, 1994; Lasanthi-Kudahettige et al., 2007; Magneschi et al., 2009; Kretzschmar et al., 2015). But
A

\begin{tabular}{|c|c|c|c|}
\hline & seq-rs2699 & seq-rs2701 & Numbers \\
\hline Hap.A & CC & TT & 198 \\
\hline Hap.B & TC & CC & 214 \\
\hline
\end{tabular}

B

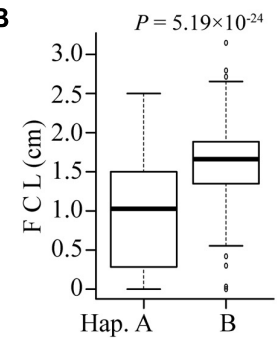

\section{c}

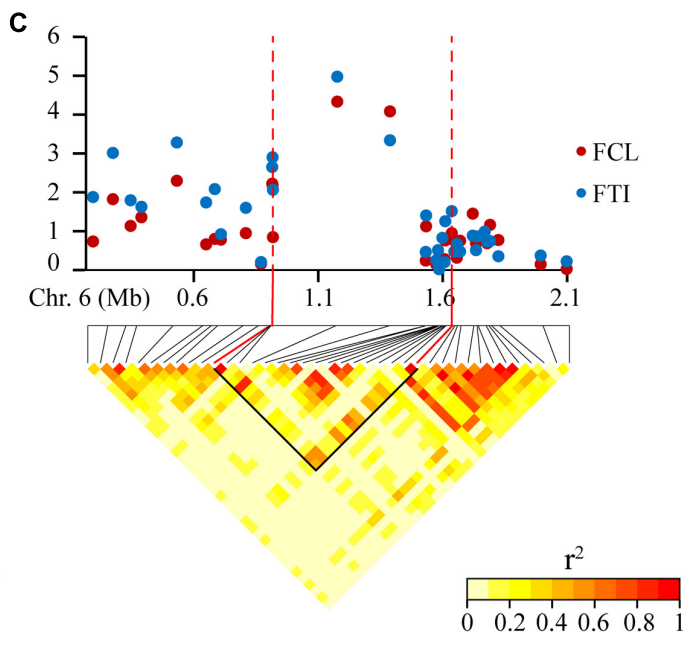

FIGURE 3 | Statistical analysis and candidate region estimation of seq-rs2699 and seq-rs2701. (A) Haplotypes consist of the two significant SNPS, Numbers indicates the amounts of corresponding accessions; (B) Phenotypic effect of each haplotype; (C) Local manhattan plots and LD heatmap around the peak on chromosome 6 , the candidate region estimated using $r^{2}>0.6$. 


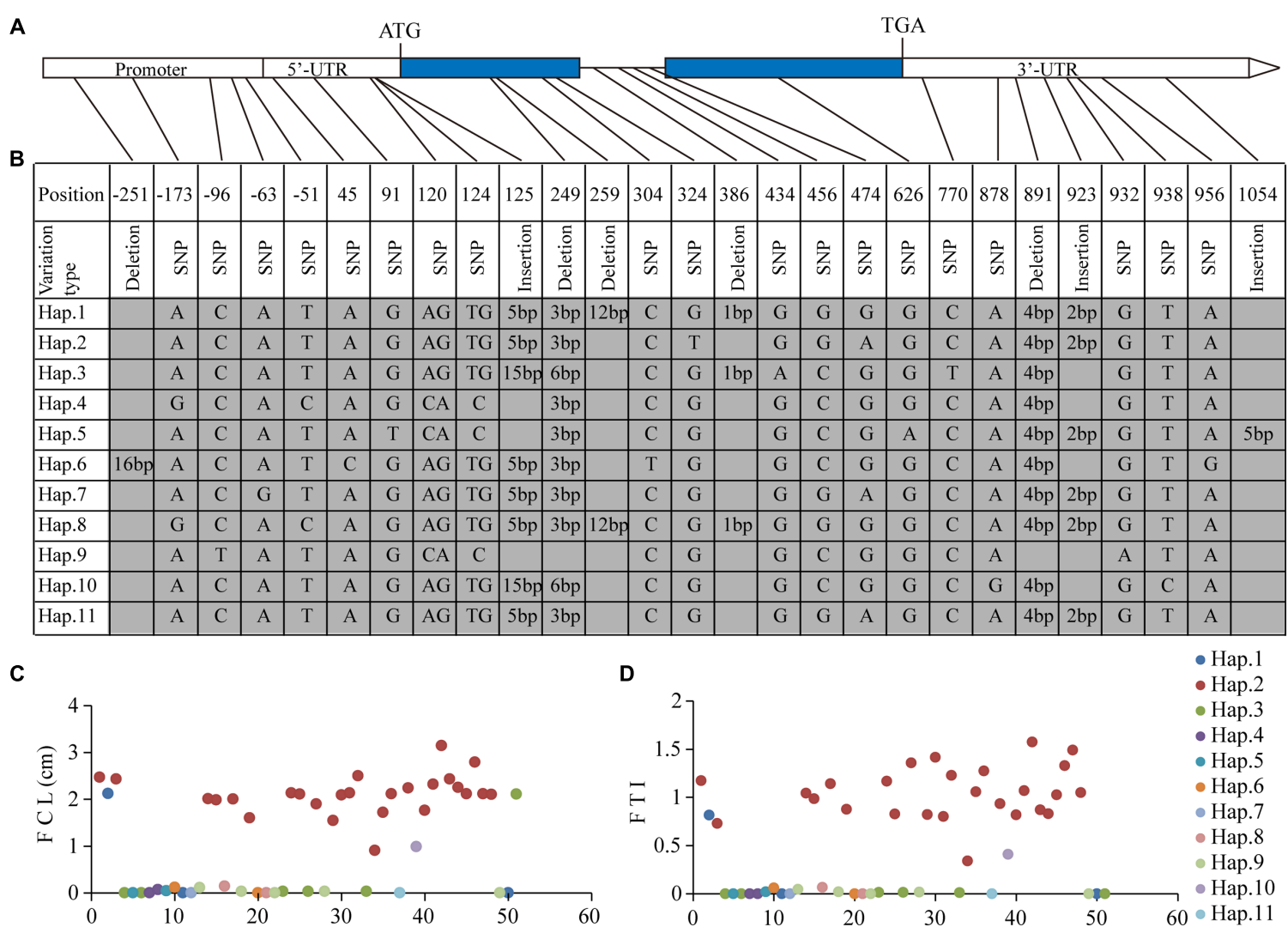

FIGURE 4 | Analysis of candidate gene LOC_Os06g03520. (A) Gene structure of LOC_Os06g03520, the blue regions show the exons; (B) eleven haplotypes detected in 51 varieties with extreme phenotypes; (C,D) phenotypic distribution of the 51 varieties, $\mathbf{x}$-axis indicates numbers sort by accessions ID; $y$-axis represents phenotypes. (C) FCL, flooded coleoptile length, (D) FTI, flooding tolerance index.

few studies took variety characteristics into consideration. In this study, we found not all of the indica varieties respond to flooding by elongating coleoptiles rapidly. Some varieties displayed suppressed FCL compared with NCL. It can be inferred the molecular mechanism of these two responses were totally different. Our results of low correlation between NCL and FCL indicated flooding had triggered response and caused phenotypic changes. This demonstrated coleoptile elongation was strongly influenced by flooding. We tried to analyze the components inside coleoptile length. We assumed FCL was constituted of two components, the original part and the part induced by flooding. If we plan to study the response to flooding, the original part should be divided. However, the variation of coleoptile observed in normal environment was extremely low, which meant the part induced by flooding accounted for the majority of phenotypic variation. The common signals detected with FCL and FTI verified that they functioned similarly. Combine with the phenotypic variation analysis and the compute mode of FTI (FCL/NCL), we concluded that both FCL and FTI could equally represent rice response level to flooding.

\section{Known Genes Identified by GWAS}

According to the results of GWAS, we have detected some SNPs close to known genes. For example, seq-rs3970 was localized close to the widely known oxygen-deficiency related gene - OsRAMY3D. The major function of OsRAMY3D is to promote starch amylolysis under energy deficiency (Setter, 1994; Gibbs et al., 2000; Magneschi and Perata, 2009; Nagai et al., 2010). The possible connection between OsRAMY3D and flooded germination tolerance can be inferred as wellaccording to our results. Kretzschmar et al. (2015) reported OsTPP7 was involved in rice aneroarc tolerance. In tolerant type, OsTPP7 accelerates coleoptile eglongation in oxygen-deficiency environment. We identified a sigificant SNP (seq-rs4216) close to OsTPP7 at a distance of 1.4 MB. Because many SNPs around this gene were still not detected, we inferred that local LD might cause the misleading assocation and lead to the large distance.

\section{Idendified a Novel Candidate Gene Controlling Rice Flooding Tolerance}

The differential expression profile strategy was an important approach to study genes associated with a given trait (Lamb et al., 2006). We took advantage of the reported expression profile data (Lasanthi-Kudahettige et al., 2007) and combined that with our association results. Within a novel detected locus, we identified a special gene might control flooded germination 
tolerance. Even though the expression profile data was collected using a japonica cultivar, the significant difference was also detected in four indica cultivars (Supplementary Figure S1). The subsequent analysis demonstrated our inference to be correct. The consistence of gene sequence and phenotype confrimed LOC_Os06g03520 was the most likely candidate. Unlike most genes controlling anerobic germnation was annoated to involve in energy metabolism pathway (OsTPP7 and OsCIPK15), LOC_Os06g03520 was annoated as DUF domain containing protein. Untill now, more experiential evidence was required to verify its molecular function.

\section{Improve GWAS in Isolating Controlling Genes}

Since its first report, GWAS has been widely carried out in rice genetic researches. Most studies were unable to identify candidate genes because of the large LD decay distance in rice. In our study, we used a gene chip carried 5291 SNP markers which was much less than those in other researches. Based on the consequence of Lu et al. (2015), most inter-marker distances were less than $100 \mathrm{~kb}$, which was enough to cover the LD decay region. Recently, association mapping has been developed to isolated genes controling rice agronomic traits such as awn distribution, grain size, heading date, panicle number per plant (Si et al., 2016; Yano et al., 2016). The efficiency was proved to be amazingly high when Japanese scholars reported four novel genes using a signle GWAS (Yano et al., 2016). Because the denisty of our SNPs was not large enough to cover every candidate gene, we add sequence comparison of numerous individuals and expression profile analysis to confrim the result. We belive RNA-seq or transcriptome chip would be good techniques since they can

\section{REFERENCES}

Alpi, A., and Beevers, H. (1983). Effects of $\mathrm{O}_{2}$ concentration on rice seedlings. Plant Physiol. 71, 30-34. doi: 10.1104/pp.71.1.30

Angaji, S. A. (2008). Mapping QTLs for submergence tolerance during germination in rice. Afr. J. Biotechnol. 7, 2551-2558. doi: 10.1007/s00122-0132057-1

Angaji, S. A., Septiningsih, E. M., Mackill, D. J., and Ismail, A. M. (2009). QTLs associated with tolerance of flooding during germination in rice (Oryza sativa L.). Euphytica 172, 159-168. doi: 10.1007/s10681-009-0014-5

Batistic, O., and Kudla, J. (2004). Integration and channeling of calcium signaling through the CBL calcium sensor/CIPK protein kinase network. Planta 219, 915-924. doi: 10.1007/s00425-004-1333-3

Bradbury, P. J., Zhang, Z., Kroon, D. E., Casstevens, T. M., Ramdoss, Y., and Buckler, E. S. (2007). TASSEL: software for association mapping of complex traits in diverse samples. Bioinformatics 23, 2633-2635. doi: 10.1093/ bioinformatics/btm 308

Chen, P. W., Chiang, C. M., Tseng, T. H., and Yu, S. M. (2006). Interaction between rice MYBGA and the gibberellin response element controls tissuespecific sugar sensitivity of alpha-amylase genes. Plant Cell 18, 2326-2340. doi: $10.1105 /$ tpc. 105.038844

Fukao, T., and Bailey-Serres, J. (2008). Submergence tolerance conferred by Sub1A is mediated by SLR1 and SLRL1 restriction of gibberellin responses in rice. Proc. Natl. Acad. Sci. U.S.A. 105, 16814-16819. doi: 10.1073/pnas.0807821105

Fukao, T., Xu, K., Ronald, P. C., and Bailey-Serres, J. (2006). A variable cluster of ethylene response factor-like genes regulates metabolic and developmental acclimation responses to submergence in rice. Plant Cell 18, 2021-2034. doi: 10.1105/tpc. 106.043000 guide researchers to a specific gene located in the association region.

\section{AUTHOR CONTRIBUTIONS}

MZ, QL, and XW: designed the research experiments. MZ, QL, and XN: performed the phenotyping. QX, YF, and CW: carried out the genetic studies. WW, XY, HY, and YW: managed the materials. XW: designed the overall project. MZ and QL: analyzed the data and drafted the manuscript. SW and CW: helped to revise the manuscript. All of the authors read and approved the final manuscript.

\section{ACKNOWLEDGMENTS}

We sincerely thank all the participants for their contribution. This work was supported by the Ministry of Science and Technology of China (2016YFD0100101-02) and the Chinese Academy of Agricultural Sciences (CAAS-ASTIP-201X-CNRRI).

\section{SUPPLEMENTARY MATERIAL}

The Supplementary Material for this article can be found online at: http://journal.frontiersin.org/article/10.3389/fpls.2017.00678/ full\#supplementary-material

FIGURE S1 | Relative expression level of LOC_Os06g03520 detected in four indcia varieties and a japonica variety Nipponbare (NPB). (A) Expression level in coleoptiles after germination for 2 days. (B) Expression level in coleoptiles after germination for 6 days.

Gibbs, J., Morrell, S., Valdez, A., Setter, T., and Greenway, H. (2000). Regulation of alcoholic fermentation in coleoptiles of two rice cultivars differing in tolerance to anoxia. J. Exp. Bot. 51, 785-796. doi: 10.1093/jxb/51.345.785

Halford, N. G., Hey, S., Jhurreea, D., Laurie, S., McKibbin, R. S., Paul, M., et al. (2003). Metabolic signalling and carbon partitioning: role of Snf1-related (SnRK1) protein kinase. J. Exp. Bot. 54, 467-475. doi: 10.1093/jxb/erg038

Hsu, S. K., and Tung, C. W. (2015). Genetic mapping of anaerobic germinationassociated QTLs controlling coleoptile elongation in rice. Rice 8, 38. doi: 10.1186/s12284-015-0072-3

Huang, S., Greenway, H., and Colmer, T. D. (2003). Anoxia tolerance in rice seedlings: exogenous glucose improves growth of an anoxia- 'intolerant', but not of a 'tolerant'genotype. J. Exp. Bot. 54, 2363-2373. doi: 10.1093/jxb/erg252

Huang, X., Wei, X., Sang, T., Zhao, Q., Feng, Q., Zhao, Y., et al. (2010). Genomewide association studies of 14 agronomic traits in rice landraces. Nat. Genet. 42 , 961-967. doi: 10.1038/ng.695

Jiang, L., Liu, S., Hou, M., Tang, J., Chen, L., Zhai, H., et al. (2006). Analysis of QTLs for seed low temperature germinability and anoxia germinability in rice (Oryza sativa L.). Field Crops Res. 98, 68-75. doi: 10.1016/j.fcr.2005. 12.015

Kang, H. M., Zaitlen, N. A., Wade, C. M., Kirby, A., Heckerman, D., Daly, M. J., et al. (2008). Efficient control of population structure in model organism association mapping. Genetics 178, 1709-1723. doi: 10.1534/genetics. 107.080101

Kato-Noguchi, H., and Morokuma, M. (2007). Ethanolic fermentation and anoxia tolerance in four rice cultivars. J. Plant Physiol. 164, 168-173. doi: 10.1016/j. jplph.2005.09.017

Kretzschmar, T., Pelayo, M. A. F., Trijatmiko, K. R., Gabunada, L. F. M., Alam, R., Jimenez, R., et al. (2015). A trehalose-6-phosphate phosphatase enhances 
anaerobic germination tolerance in rice. Nat. Plants 1:15124. doi: 10.1038/ nplants.2015.124

Kudahettige, N. P., Pucciariello, C., Parlanti, S., Alpi, A., and Perata, P. (2011). Regulatory interplay of the Sub1A and CIPK15 pathways in the regulation of alpha-amylase production in flooded rice plants. Plant Biol. 13, 611-619. doi: 10.1111/j.1438-8677.2010.00415.x

Lamb, J., Crawford, E. D., Peck, D., Modell, J. W., Blat, I. C., Wrobel, M. J., et al. (2006). The connectivity map: using gene-expression signatures to connect small molecules. Genes Dis. Sci. 313, 1929-1935. doi: 10.1126/science.1132939

Lasanthi-Kudahettige, R., Magneschi, L., Loreti, E., Gonzali, S., Licausi, F., Novi, G., et al. (2007). Transcript profiling of the anoxic rice coleoptile. Plant Physiol. 144, 218-231. doi: 10.1104/pp.106.093997

Lee, K. W., Chen, P. W., Lu, C. A., Chen, S., Ho, T. D., and Yu, S. M. (2009). Coordinated responses to oxygen and sugar deficiency allow rice seedlings to tolerate flooding. Sci. Signal. 2, ra61. doi: 10.1126/scisignal.2000333

Librado, P., and Rozas, J. (2009). DnaSP v5: a software for comprehensive analysis of DNA polymorphism data. Bioinformatics 25, 1451-1452. doi: 10.1093/ bioinformatics/btp187

Liu, K., and Muse, S. V. (2005). PowerMarker: an integrated analysis environment for genetic marker analysis. Bioinformatics 21, 2128-2129. doi: 10.1093/ bioinformatics/bti282

Loreti, E., Poggi, A., Novi, G., Alpi, A., and Perata, P. (2005). A genome-wide analysis of the effects of sucrose on gene expression in Arabidopsis seedlings under anoxia. Plant Physiol. 137, 1130-1138. doi: 10.1104/pp.104.057299

Lu, C. A. (2002). Three novel MYB proteins with one DNA binding repeat mediate sugar and hormone regulation of alpha-amylase gene expression. Plant Cell 14, 1963-1980. doi: 10.1105/tpc.001735

Lu, C. A., Lin, C. C., Lee, K. W., Chen, J. L., Huang, L. F., Ho, S. L., et al. (2007). The SnRK1A protein kinase plays a key role in sugar signaling during germination and seedling growth of rice. Plant Cell 19, 2484-2499. doi: 10.1105/tpc.105. 037887

Lu, Q., Zhang, M., Niu, X., Wang, S., Xu, Q., Feng, Y., et al. (2015). Genetic variation and association mapping for 12 agronomic traits in indica rice. $B M C$ Genomics 16:1067. doi: 10.1186/s12864-015-2245-2

Magneschi, L., Kudahettige, R. L., Alpi, A., and Perata, P. (2009). Comparative analysis of anoxic coleoptile elongation in rice varieties: relationship between coleoptile length and carbohydrate levels, fermentative metabolism and anaerobic gene expression. Plant Biol. 11, 561-573. doi: 10.1111/j.1438-8677. 2008.00150.x

Magneschi, L., and Perata, P. (2009). Rice germination and seedling growth in the absence of oxygen. Ann. Bot. 103, 181-196. doi: 10.1093/aob/mcn121

Nagai, K., Hattori, Y., and Ashikari, M. (2010). Stunt or elongate? Two opposite strategies by which rice adapts to floods. J. Plant Res. 123, 303-309. doi: 10.1007/s10265-010-0332-7

Rohlf, F. J. (ed.). (2000). "Numerical taxonomy and multivariate analysis system," in Exeter Software Version 2, 1 Edn, (New York: Rohlf FJ).

Sedbrook, J. C., Kronebusch, P. J., Borisy, G. G., Trewavas, A. J., and Masson, P. H. (1996). Transgenic AEQUORIN reveals organ-specific cytosolic $\mathrm{Ca}^{2+}$ responses to anoxia in Arabidopsis thaliana seedlings. Plant Physiol. 111, 243257. doi: $10.1104 /$ pp.111.1.243
Septiningsih, E. M., Ignacio, J. C., Sendon, P. M., Sanchez, D. L., Ismail, A. M., and Mackill, D. J. (2013). QTL mapping and confirmation for tolerance of anaerobic conditions during germination derived from the rice landrace Ma-Zhan Red. Theor. Appl. Genet. 126, 1357-1366. doi: 10.1007/s00122-0132057-1

Septiningsih, E. M., Pamplona, A. M., Sanchez, D. L., Neeraja, C. N., Vergara, G. V., Heuer, S., et al. (2009). Development of submergence-tolerant rice cultivars: the Sub1 locus and beyond. Ann. Bot. 103, 151-160. doi: 10.1093/aob/ men206

Setter, T. (1994). Relationship between coleoptile elongation and alcoholic fermentation in rice exposed to anoxia. I. importance of treatment conditions and different tissues. Ann. Bot. 74, 265-271. doi: 10.1006/anbo.1994.1117

Shin, J.-H., Blay, S., McNeney, B., and Graham, J. (2006). LDheatmap: an R function for graphical display of pairwise linkage disequilibria between single nucleotide polymorphisms. J. Stat. Softw. 3, 1-10. doi: 10.18637/jss.v016.c03

Si, L., Chen, J., Huang, X., Gong, H., Luo, J., Hou, Q., et al. (2016). OsSPL13 controls grain size in cultivated rice. Nat. Genet. 48, 447-456. doi: 10.1038/ng. 3518

Subbaiah, C. C., Zhang, J., and Sachs, M. M. (1994). Involvement of intracellular calcium in anaerobic gene expression and survival of maize seedlings. Plant Physiol. 105, 369-376. doi: 10.1104/pp.105.1.369

Tamura, K., Stecher, G., Peterson, D., Filipski, A., and Kumar, S. (2013). MEGA6: molecular evolutionary genetics analysis version 6.0. Mol. Biol. Evol. 30, 2725-2729. doi: 10.1093/molbev/mst197

Wen, Z., Tan, R., Yuan, J., Bales, C., Du, W., Zhang, S., et al. (2014). Genome-wide association mapping of quantitative resistance to sudden death syndrome in soybean. BMC Genomics 15:809. doi: 10.1186/1471-2164-15-809

$\mathrm{Xu}, \mathrm{K} ., \mathrm{Xu}, \mathrm{X}$. , Fukao, T., Canlas, P., Maghirang-Rodriguez, R., Heuer, S., et al. (2006). Sub1A is an ethylene-response-factor-like gene that confers submergence tolerance to rice. Nature 442, 705-708. doi: 10.1038/nature 04920

Yano, K., Yamamoto, E., Aya, K., Takeuchi, H., Lo, P. C., Hu, L., et al. (2016). Genome-wide association study using whole-genome sequencing rapidly identifies new genes influencing agronomic traits in rice. Nat. Genet. 48, 927-934. doi: 10.1038/ng.3596

Zhang, Z., Ersoz, E., Lai, C. Q., Todhunter, R. J., Tiwari, H. K., Gore, M. A., et al. (2010). Mixed linear model approach adapted for genome-wide association studies. Nat. Genet. 42, 355-360. doi: 10.1038/ng.546

Conflict of Interest Statement: The authors declare that the research was conducted in the absence of any commercial or financial relationships that could be construed as a potential conflict of interest.

Copyright (c) 2017 Zhang, Lu, Wu, Niu, Wang, Feng, Xu, Wang, Yuan, Yu, Wang and Wei. This is an open-access article distributed under the terms of the Creative Commons Attribution License (CC BY). The use, distribution or reproduction in other forums is permitted, provided the original author(s) or licensor are credited and that the original publication in this journal is cited, in accordance with accepted academic practice. No use, distribution or reproduction is permitted which does not comply with these terms. 